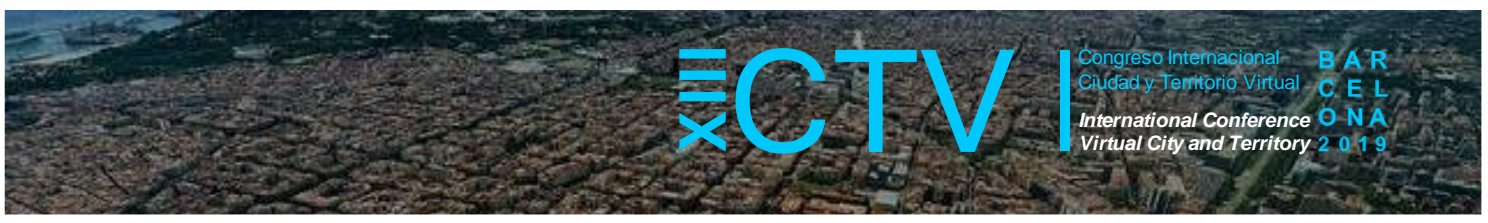

\title{
ESTRATEGIA PARA LA EXTRACCIÓN DEL CONOCIMIENTO COMO APOYO EN LA ELABORACIÓN DE LOS PLANEAMIENTOS URBANÍSTICOS
}

\author{
Ramírez-Uribe, Gerardo ${ }^{1 *}$; Quintana, Jesús ${ }^{2}$; Ojeda, Arturo ${ }^{3}$ y Silvestre, José ${ }^{4}$
}

Remisión inicial: 2019-04-29; Remisión definitiva: 2019-09-09; Publicación: 2019-12-21

Citación: Ramírez-Uribe, G. et al. (2019). Estrategia para la extracción del conocimiento como apoyo en la elaboración de los planeamientos urbanísticos. En XIII CTV 2019 Proceedings: XIII International Conference on Virtual City and Territory: "Challenges and paradigms of the contemporary city": UPC, Barcelona, October 2-4, 2019. Barcelona: CPSV, 2019, p. 8261. E-ISSN 2604-6512. DOI http://dx.doi.org/10.5821/ctv.8261

\section{Resumen}

La presente investigación se basa en gestión de conocimiento (GC), para desarrollar una estrategia como soporte en la elaboración de planes y proyectos a escala de barrio o mejora de centro histórico. La estrategia se ha implementado en el casco antiguo de San Andreu en Barcelona y parte de los resultados revelaron la necesidad de aprovechar el conocimiento que poseen los gestores/planificadores urbanos en temas de planificación urbana. Se conceptualiza una estrategia para que, en el cual es viable el planear, desarrollar e implementar la GC en temas de planeamiento urbanístico. Esta estrategia consta de tres fases: la primera el análisis de la estrategia, esta se compone de tres etapas: el inventario de conocimiento, el mapa de conocimiento y el análisis de la red, la segunda fase: el análisis FODA y la última fase: el sistema tecnológico. La información que se obtiene de las entrevistas a profundidad es una base para determinar el inventario de conocimiento y así, se identifica quiénes son los titulares de los conocimientos fundamentales. La información obtenida de las entrevistas está organizada en los inventarios de conocimientos para identificar quiénes son los titulares de los conocimientos fundamentales. Para el desarrollo del proyecto se realiza la recogida de datos y extracción del conocimiento de uno de los ejes importantes y necesarios para la elaboración del planeamiento, la visión del planificador / gestor de planes y proyectos para la mejora de barrios o centros históricos. Dicha estrategia, propone la extracción del conocimiento necesario para la elaboración de los planeamientos urbanísticos a escala de barrio o mejora de centro histórico, aquí se considerará una combinación de herramientas y algunas mezclas de técnicas de diseño del sistema basado en el conocimiento. La estrategia, tiene como objetivo de visualizar la generación/transferencia del conocimiento de los tres ejes importantes (planificador/gestor, habitantes del ámbito de estudio y morfología urbana) necesarios para la elaboración del planeamiento. De esta etapa se concluye que los principales logros o avances en el conocimiento obtenidos en tema de sistema de GC orientando al planeamiento urbanístico, resaltan que los planificadores/gestores disponen de información básica en línea desde software informático (Vista, Vissir, catastro, padrón, etc.), pero algunos de ellos, tal como, carecen de lo social o de la percepción de los habitantes del barrio, también es posible destacar, que el factor tiempo de los agentes es impedimento para el proceso de la ejecución. El análisis FODA facilitó la comprensión de las fortalezas y debilidades, ayuda a clarificar y resumir las principales cuestiones y oportunidades de la información obtenida, y esto facilita el desarrollo del pensamiento estratégico, permitió explorar eficazmente los factores positivos y neutralizar o eliminar el efecto de los factores negativos, genera ideas nuevas para ayudar a aprovechar las fortalezas. Finalmente, toda la información generada del análisis de la estrategia y del análisis FODA de la estrategia fue guardada en el sistema tecnológico para su fácil gestión y utilización de la misma. Este documento debate la relevancia de la utilización de metodologías de GC en la elaboración de planes de mejora urbana, que, aunque es un tema en auge, existe evidencia dentro del sector empresarial u organizacional que demuestra que la GC es un instrumento que aporta valor añadido en temas relacionados a este sector. Esta investigación ha mostrado el uso de la planificación estratégica colaborativa para la resolución de problemas de planificación urbana a escala de barrio o mejora de centro histórico, siendo esta de gran importancia, por lo cual, es posible avalar que las GC, debido a los resultados mostrados en este documento, que aunque su mayor aplicación en un sector con distinta problemática, pueden aportar información importante en la resolución de problemas urbanísticos, tal y como sucede con la planeación estratégica.

\footnotetext{
${ }^{1}$ Dr. Ingeniero, Universidad de Sonora, http://orcid.org/0000-0001-7036-764X, ${ }^{2}$ Dr. Ingeniero, Universidad de Sonora, http://orcid.org/0000-0003-1797-6213, ${ }^{3}$ Dr. Ingeniero, Universidad de Sonora, http://orcid.org/0000-0002-4513-514X y ${ }^{4}$ Dr. Ingeniero, Universidad de Sonora. * Correo de contacto: gerardo.ramirez@unison.mx
} 


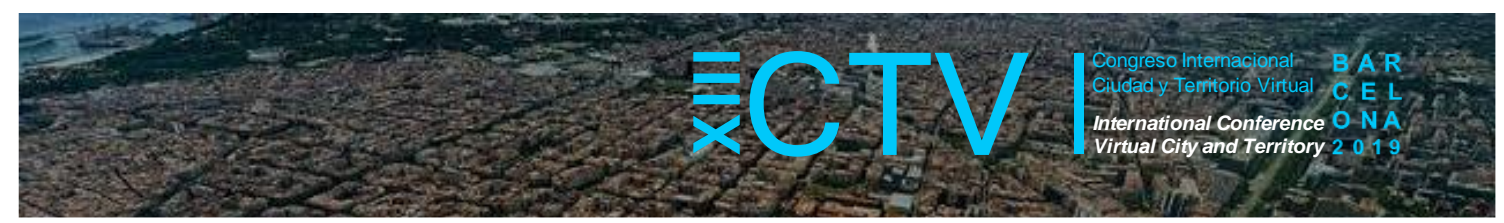

\section{Abstract}

This research is based on knowledge management (KM), to develop a strategy to support the development of plans and projects at the neighborhood level or improvement of the historic center. The strategy has been implemented in the old town of San Andreu in Barcelona and part of the results revealed the need to take advantage of the knowledge that urban managers / planners possess in urban planning issues. A strategy is conceptualized so that, in which it is feasible to plan, develop and implement the KM in urban planning issues. This strategy consists of three phases: the first, the analysis of the strategy, this is composed of three stages: the knowledge inventory, the knowledge map and the network analysis, the second phase: the SWOT analysis and the last phase: The technological system. The information obtained from in-depth interviews is a basis for determining the knowledge inventory and thus, who is the holder of the fundamental knowledge is identified. The information obtained from the interviews is organized in the knowledge inventories to identify who are the holders of the fundamental knowledge. For the development of the project, data collection and knowledge extraction of one of the important and necessary axes for the preparation of the planning, the vision of the planner / manager of plans and projects for the improvement of neighborhoods or historical centers is carried out. This strategy proposes the extraction of the necessary knowledge for the development of urban planning at the neighborhood level or improvement of the historic center, here a combination of tools and some mixtures of knowledge-based system design techniques will be considered. The strategy aims to visualize the generation / transfer of knowledge of the three important axes (planner / manager, inhabitants of the field of study and urban morphology) necessary for the development of planning. From this stage, it is concluded that the main achievements or advances in knowledge obtained in terms of the KM system oriented to urban planning, highlight that the planners / managers have basic information online from computer software (Vista, Vissir, cadastre, registry, etc.), but some of them, such as, lack the social or the perception of the inhabitants of the neighborhood, it is also possible to emphasize that the time factor of the agents is an impediment to the execution process. The SWOT analysis facilitated the understanding of strengths and weaknesses, helps clarify and summarize the main issues and opportunities of the information obtained, and this facilitates the development of strategic thinking, allowed to effectively explore positive factors and neutralize or eliminate the effect of Negative factors, generates new ideas to help harness strengths. Finally, all the information generated from the strategy analysis and the SWOT analysis of the strategy was stored in the technological system for its easy management and use. This document discusses the relevance of the use of GC methodologies in the development of urban improvement plans, which, although it is a growing issue, there is evidence within the business or organizational sector that demonstrates that the KM is an instrument that provides added value on issues related to this sector. This research has shown the use of collaborative strategic planning for the resolution of urban planning problems at the neighborhood level or improvement of the historic center, which is of great importance, therefore, it is possible to guarantee that the KM, due to the results shown in this document, which although its greater application in a sector with different problems, can provide important information in solving urban problems, as is the case with strategic planning.

Palabras Clave: Gestión de conocimiento; Morfología Urbana; Planeamiento Urbanístico; urbanismo

Key words: Knowledge management; Urban Morphology; Urban Planning; urbanism

\section{Introducción}

Egbu y Botterill (2002) interpretaron la gestión del conocimiento (GC) como los 'procesos mediante los cuales el conocimiento se crea, se adquiere, se comunica, se comparte, se aplica y se utiliza y administra efectivamente, para satisfacer las necesidades existentes y emergentes, para identificar y explotar los conocimientos existentes y adquiridos. Vetzal (2005), entiende que los datos representan hechos y cifras descontextualizados, la información como datos organizados o contextualizados. Matsumoto (2005) enfatizó que el principal medio para desarrollar conocimiento fue a través de su adquisición a través del tiempo a través de la experiencia.

La gestión del conocimiento comprende una gama de estrategias y prácticas utilizadas en una organización para identificar, crear, representar, distribuir y permitir la adopción de experiencias, mejores prácticas, lecciones, procesos, tecnologías e información. Este conocimiento puede estar incorporado en individuos o incrustado en procesos organizativos 0 en la práctica. La GC va más allá de la gestión de la información porque proporciona 


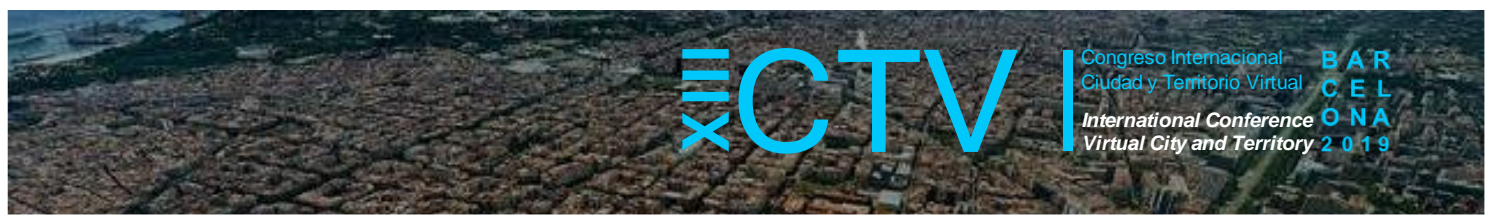

herramientas para promover el intercambio y la colaboración. En el contexto de UN-HABITAT (2010), la GC busca apoyar el intercambio de información relevante y conocimiento entre el personal para la efectividad organizacional; y aprovechar los esfuerzos de los socios del Programa de Hábitat para lograr una urbanización sostenible.

El intercambio de conocimientos sigue siendo un tema difícil. Incluso dentro del gobierno, el conocimiento no viaja bien entre los departamentos y los diferentes niveles de gobierno. El intercambio de conocimiento es escaso y en algunos contextos existe una resistencia activa a la digitalización de las bases de datos internas debido al temor de que una mayor transparencia y control revelen incompetencia y corrupción. Sin embargo, se están emprendiendo algunas iniciativas en esta área. El intercambio de conocimientos y la retroalimentación de los residentes generalmente está limitado y se limita a pautas estrictas. Sin embargo, los temas de contra mapeo en otros lugares se están abriendo a través de las ONG (Baud, 2016).

En otra investigación realizada por el Instituto Penn para la Investigación Urbana, el cual, está llevando a cabo un estudio de paisaje sobre cómo las ciudades transfieren conocimiento en áreas de desarrollo urbano sostenible. A medida que el modelo de "Mejores Prácticas" continúa consolidando su lugar en el centro de un número creciente de disciplinas, desde el desarrollo tecnológico del sector privado hasta la gobernanza y la participación cívica, ha habido un aumento simultáneo en la atención de especialistas urbanos, profesionales y académicos. de la misma manera: cómo se desarrolla y difunde el conocimiento en las metrópolis del mundo (Birch, 2011). El estudio realizado por las principales organizaciones internacionales, como el Banco Mundial (Chen y Dahlman, 2005), la Comisión Europea (Morgan, 2007), las Naciones Unidas (Juma y Yee-Cheong, 2005) y la Organización para la Cooperación y el Desarrollo Económicos (OCDE), (Cooke y Leydesdorff, 2006), han adoptado marcos de GC en sus orientaciones estratégicas con respecto al desarrollo global. Esta serie de estrategias indica la fuerza del vínculo que ha surgido entre el conocimiento, la GC y el desarrollo urbano (Komninos, 2002; Ergazakis et al., 2004). Malizia (2015), propone una metodología la cual tiene por objetivo presentar una estrategia metodológica para el estudio de las urbanizaciones y esta se enmarca en una investigación cualitativa, la cual permite reconstruir teóricamente un objeto en permanente dinamismo y obtener una comprensión holística de las situaciones sociales.

Existen dos tipos de conocimiento: tácito y explicito, el primero es el conocimiento difícil de articular y también es difícil de poner en palabras, texto o dibujos. El tácito tiende a residir dentro de las cabezas de los conocedores, el conocimiento explicito, por lo contrario, representa el contenido que ha sido capturado en alguna forma tangible, como palabras, grabaciones de audio o imágenes. La GC representa un enfoque deliberado y sistemático para garantizar la plena utilización de la base de conocimientos de una organización, junto con el potencial de las habilidades, competencias individuales, los pensamientos, las innovaciones y las ideas para crear una organización más eficiente y eficaz. (Dalkir, 2017).

Las diferentes formas de conocimiento espacial (conocimiento experto, tácito, sectorial y comunitario) son un recurso estratégico en el desarrollo urbano. Los métodos de investigación relacionados con la recopilación y el análisis de datos participativos que obtienen e integran las diversas formas de conocimiento o coproducen conocimiento a través de la colaboración entre académicos y profesionales tienen el potencial de informar la acción local y las políticas públicas (Pfeffer, 2010). 


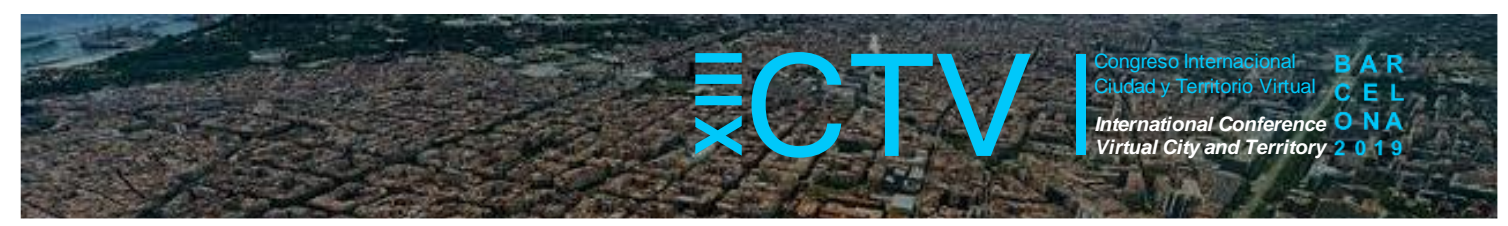

La auditoría del conocimiento es la primera y más importante etapa de una iniciativa de GC. Permite hacer una investigación y conocer el estado actual del conocimiento en la elaboración de planeamiento urbanístico, por lo tanto, es una herramienta para descubrir, verificar y validar; que provee descubrimiento de hechos, análisis, interpretación e informes. Incluye un estudio de la información corporativa, prácticas y políticas de conocimiento de la estructura y flujo de la información y conocimiento. La auditoría del conocimiento examina los recursos de conocimiento y su uso: cómo y porqué se adquiere, accede, disemina, comparte y usa el conocimiento (Aviv et al, 2009).

Mediante la auditoria del conocimiento es posible adquirir información estratégica tanto de la administración como de los habitantes del barrio e identificar los procesos en la elaboración del planeamiento, identificar los procesos básicos y establecer los criterios de medición, priorizar y seleccionar los procesos básicos, identificar las personas clave, la obtención de inventario de los conocimientos, el análisis de flujo de conocimiento; mapeo del conocimiento; los informes de auditoría del conocimiento, esta consiste en realizar: flujos de conocimiento, inventario del conocimiento, mapas del conocimiento, y esquematizar la red social (Pérez-Soltero et al, 2006). En el inventario de conocimiento se encuentra todo el conocimiento existente para los procesos clave, cuál se utiliza, dónde se utiliza, qué rol tienen cada uno de los agentes, el "cuál" se refiere a los activos de conocimiento que contribuyen al éxito en la ejecución de los procesos; el "dónde" y "cuándo" se capturan por los descriptores de tiempo y localización de un activo de conocimiento, y el "qué rol organizacional" se refiere a los roles abstractos que participan en los procesos de elaboraciones de planes urbanísticos de barrio o mejoras de centro histórico. Estos roles pueden llevarse a cabo por diferentes agentes. Ante todo, una de las tareas que se tiene que atender es la identificación de los activos de conocimiento ( $\mathrm{Wu}, 2007)$. La determinación del flujo del conocimiento se realiza mediante la creación de mapas de conocimiento y el análisis de la red social.

Un inventario de conocimiento es una forma práctica de llegar a enfrentarse con "saber lo que sabe." Este inventario se realiza generalmente mediante la aplicación de los principios de la gestión de los recursos de información (GRI), los cuales son los siguientes:

- Identificación: ¿Qué información hay para poder realizar planeamiento urbanístico? ¿Cómo se identifican y como se codifica?

- Propiedad: ¿Quién es responsable de las entidades de información y como la coordinan (agentes responsables de la elaboración de planeamiento urbanístico)?

- Costo y valor: ¿Qué es un modelo básico para la toma de decisiones en la compra y el uso del conocimiento para la elaboración de planeamiento urbanístico?

- Desarrollo: ¿Cómo podemos aumentar el valor de la información o estimular la demanda?

- Explotación: ¿Cuál es la mejor forma de maximizar el valor de forma proactiva? (Dalkir, 2017).

El proceso de mapeo de conocimientos es relativamente sencillo. Consiste en realizar un inventario de lo que personas que intervienen en la elaboración de planeamiento urbanístico han escrito o lo que ha entrado en los sistemas de información geográfica, así como la identificación de fuentes de información externas que contienen la percepción que tienen los habitantes de su barrio. 


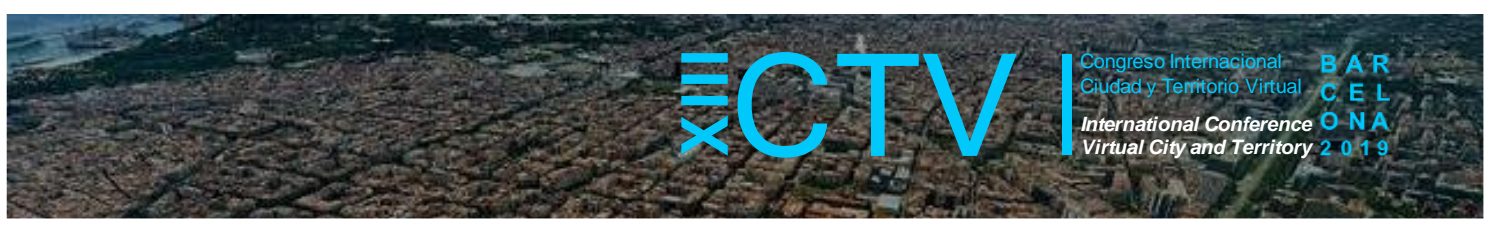

La información concentrada en el inventario de conocimiento, es luego organizada en mapas de conocimiento (MP), este es una representación del "modelo mental" de los conocimientos de una persona y proporciona una buena forma de conocimiento codificado. Un MP es una representación simbólica o cualitativa de algo en el mundo real, este es una poderosa manera de codificar este conocimiento acumulado, ya que también captura el contexto y las complejas interrelaciones entre los conceptos. De hecho, es también muy importante incluir las opiniones individuales, las percepciones, juicios, hipótesis y creencias que forman parte de la visión del mundo subjetivo del entrevistado. Los nodos en un mapa son los conceptos clave, y los enlaces que representan las relaciones entre los conceptos (Dalkir, 2017).

Para la elaboración de mapas de conocimiento se utiliza la herramienta ConceptDraw Office Mind Maps, que es una aplicación informática de gran alcance y de alta productividad personal que proporciona la organización y el acceso visual a la información para los individuos y las organizaciones, La Figura 1 presenta un ejemplo del diseño de un mapa de conocimiento con ConceptDraw MINDMAP (Concepdraw Mindmaps, 2019).

Figura 1. Diseño de un mapa de conocimiento realizado con ConceptDraw MINDMAP

Fuente: Adaptado de Software para creación de mapa de conocimiento, 2019.

La Figura 2 muestra los procedimientos para la construcción del mapa de conocimiento. El procedimiento consta de seis pasos: la definición de los conocimientos de la organización, análisis del mapa de procesos, extracción de conocimiento, el perfil de conocimientos, vinculación del conocimiento y la validación de mapa de conocimientos (Kim et al, 2003).

Figura 2. Procedimiento para la construcción de un mapa de conocimiento

Fuente: Adaptado de Kim et al, 2003.

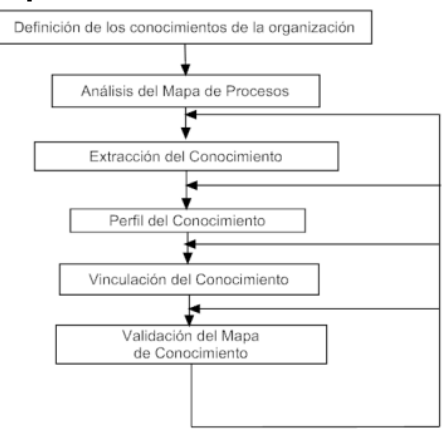

La información que se concentra en el MP se utiliza para realizar el análisis de la red social (ARS), éste resalta la transferencia de conocimiento entre los agentes, haciendo uso de sus respectivos grados de emisión, recepción y status sociométrico. Para el ARS, existe diversos softwares para realización del mismo. Uno de ellos es Netdraw (Netdraw, 2019). La Figura 3 


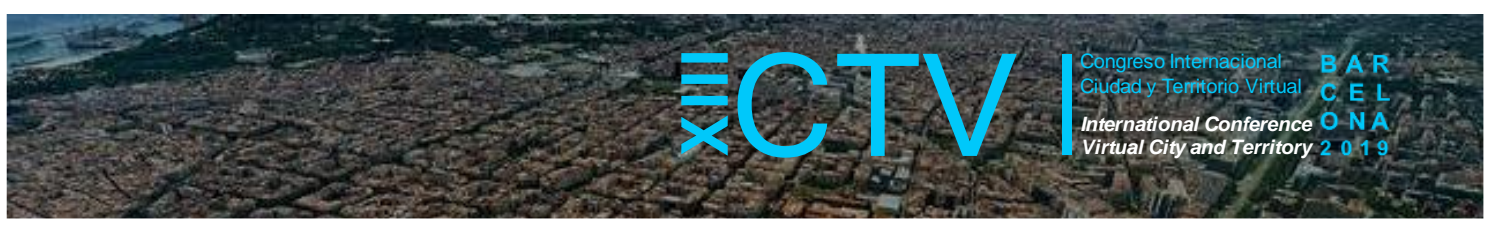

muestra el diseño de una red social construida con Netdraw, en ella se observa el flujo de conocimiento que existe entre los agentes, los perfiles de cada uno de ellos, los géneros de los agentes dependen del color de la figura, y la forma de la figura se refiere a la responsabilidad del agente (Software para GC, 2019).

Figura 3. Red Social construida con Netdraw

Fuente: Adaptado de Software para GC, 2016

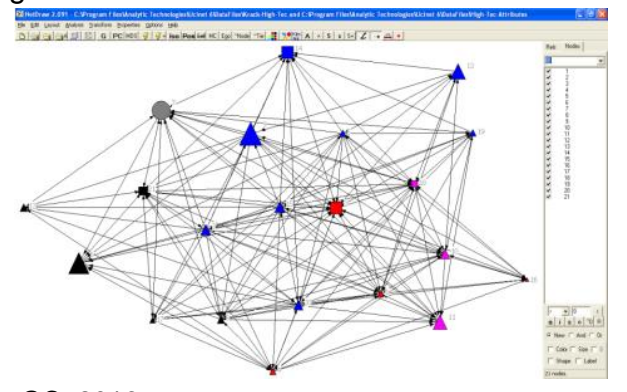

El vínculo entre los conocimientos, indica cuando se realiza la producción del perfil de conocimiento, y más tarde se confirma. Vincular el conocimiento se representa como una flecha en un mapa de conocimientos. El mapa de conocimiento muestra una ruta de navegación de los conocimientos. El mapa de conocimiento es un tipo de gráfico dirigido y se compone de nodos y enlaces, cada nodo de elemento que denota el conocimiento y la relación que denota pre y post-relación entre los conocimientos, en donde $\mathrm{Kr}$ son los nodos donde se genera el conocimiento y $\mathrm{Sr}$ es la relación que existe entre los nodos. La Figura 4 muestra un ejemplo de cómo se lleva a cabo la vinculación de conocimiento, los valores $\mathrm{K}$ se refieren al conocimiento generado, recibido o retenido, por los usuarios S, las líneas con flechas muestran la dirección o tendencias del conocimiento de los usuarios, un ejemplo de cómo fluye el conocimiento en una organización.

Figura 4. Vinculación del Conocimiento

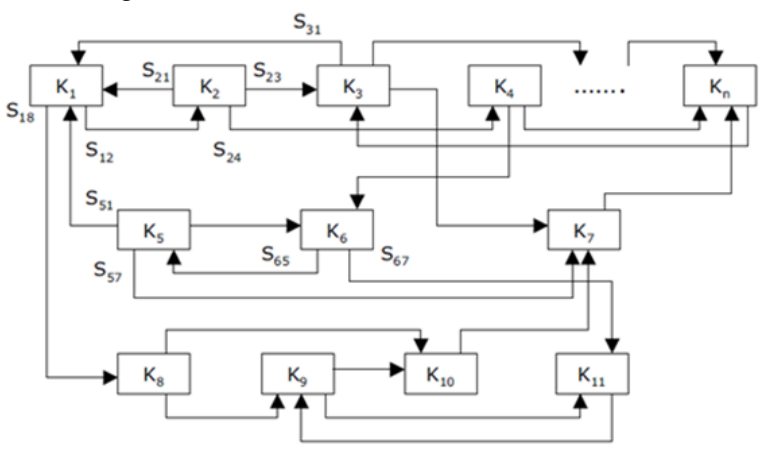

Fuente: Adaptado de Kim et al, 2003.

Es importante destacar que algunos factores tienen mayor preponderancia que otros, como lo plantea Strickland (2001), al denominar el análisis FODA como la construcción de un balance estratégico, mientras que los aspectos considerados fuertes de una organización son los activos competitivos y los débiles son los pasivos también competitivos. Pero se comete un error si se trata de equilibrar la balanza. Lo importante radica en que los activos competitivos 0 aspectos fuertes superen a los pasivos competitivos o situaciones débiles, lo que debe trascender es en darle mayor ponderación a los activos. 


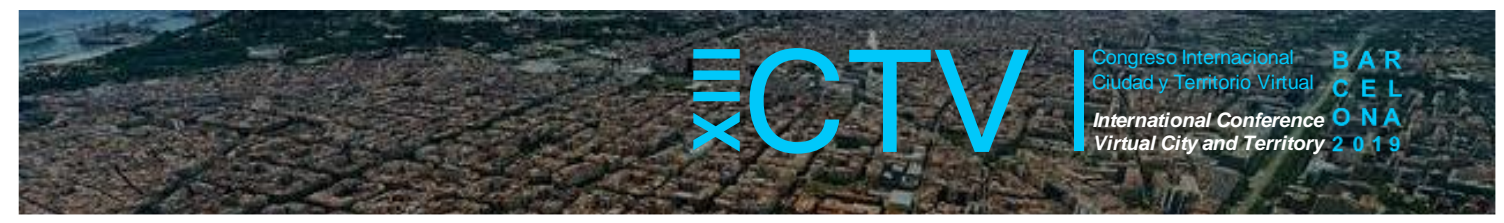

Para realizar el análisis FODA también se puede recurrir a la técnica de lluvia de ideas, con la participación de directivos y mandos clave, donde cada uno genere por separado de 5 a 10 de las principales debilidades que la organización tiene. Es posible recurrir a ciertos instrumentos que ayuden a profundizar mejor en los aspectos críticos de cada elemento del FODA, como son los estudios de desempeño de la organización, los análisis externos, las opiniones de expertos, etc. Una vez generadas las ideas del análisis, un grupo más pequeño se puede dar a la tarea de agrupar y organizar la información generada (los aspectos que reciben más menciones, etc.). Este análisis se presenta al equipo directivo para que sea enriquecido y discutido. El resultado final debe ser una relación jerarquizada de los elementos del análisis. Es conveniente que la síntesis del FODA se coloque y ordene en una matriz $2 \times 2$; en la parte superior las $\mathrm{F}$ y las $\mathrm{O}$, y en la parte inferior las D y las A (Gutiérrez, 2005).

En los últimos años, los repositorios institucionales (RI) han cobrado importancia en la sociedad académica y científica, porque representan una fuente de información digital especializada, organizada y accesible para los lectores de diversas áreas. Los RI son sistemas informáticos dedicados a gestionar los trabajos científicos y académicos de diversas instituciones de forma libre y gratuita, es decir, siguiendo las premisas del movimiento Open Access (OA).

Los repositorios están compuestos por múltiples archivos digitales representativos de la producción intelectual que resulta de la actividad investigadora de la comunidad científica y tiene la finalidad de organizarla, preservarla y difundirla en modo de acceso abierto.

Los repositorios institucionales consisten en estructuras web interoperables de servicios informáticos, dedicadas a difundir la perpetuidad de los recursos científicos y académicos (físicos o digitales) de las universidades, a partir de la enumeración de un conjunto de datos específicos (metadatos), para que esos recursos se puedan recopilar, catalogar, acceder, gestionar, difundir y preservar de forma libre y gratuita, de manera que están estrechamente ligados a los ideales y objetivos del acceso abierto (Duperet, 2015).

\section{Metodología y materiales}

Se conceptualiza un modelo para que, en el cual es viable el planear, desarrollar e implementar la GC en temas de planeamiento urbanístico. Esta estrategia consta de tres fases: la primera el análisis de la estrategia, esta se compone de tres etapas: el inventario de conocimiento, el mapa de conocimiento y el análisis de la red, la segunda fase: el análisis FODA y la última fase: el sistema tecnológico. La Figura 5 muestra la estrategia propuesta que se desarrolla en la cual se proporciona una guía detallada del sistema.

Figura 5. Estrategia para la extracción del conocimiento para la elaboración de los planeamientos urbanísticos a escala de barrio o mejora de centro histórico

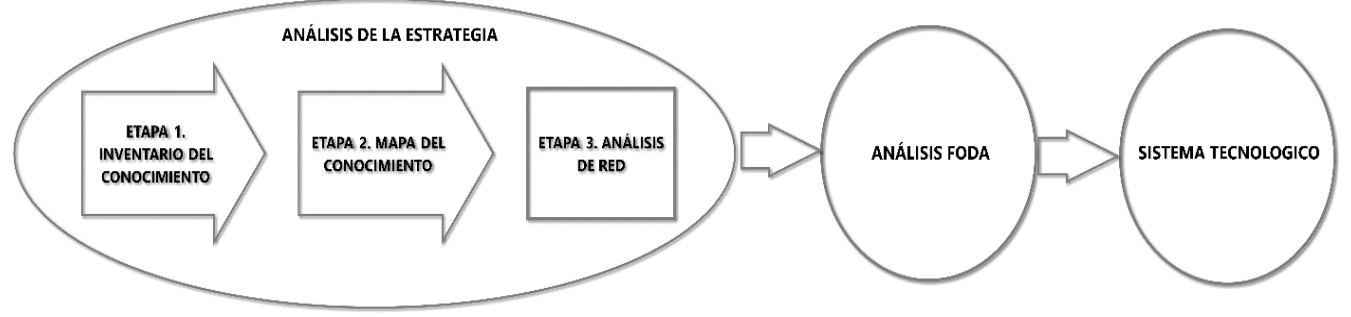

Fuente: Elaboración propia. 


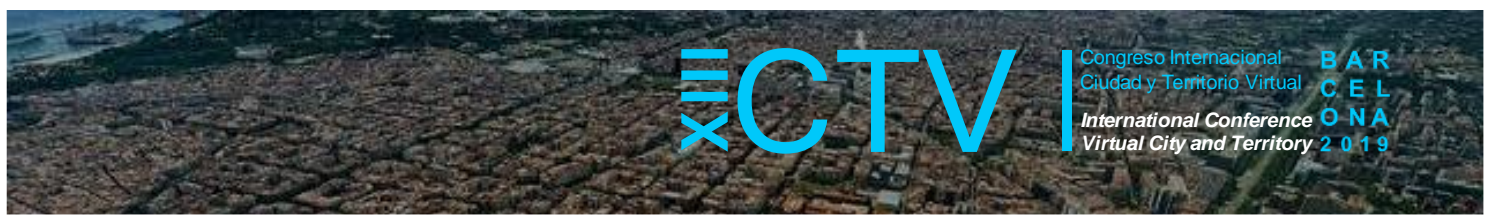

Dicha estrategia, propone la extracción del conocimiento necesario para la elaboración de los planeamientos urbanísticos a escala de barrio o mejora de centro histórico, aquí se considerará una combinación de herramientas y algunas mezclas de técnicas de diseño del sistema basado en el conocimiento, tales como las estrategias de adquisición de conocimiento estructurado por expertos en la materia, inventarios de conocimiento, mapas de conocimiento y análisis de la red social, esto se describe a continuación.

\subsection{Primera Fase}

\subsubsection{Elaboración del inventario del conocimiento}

La información que se obtiene de las entrevistas a profundidad es una base para determinar el inventario de conocimiento y así, se identifica quiénes son los titulares de los conocimientos fundamentales. La información obtenida de las entrevistas está organizada en los inventarios de conocimientos para identificar quiénes son los titulares de los conocimientos fundamentales. En Tabla 1 se puede observar parte del inventario de conocimiento y el conocimiento que se requiere para realizar las actividades en la elaboración de planeamientos urbanísticos.

Tabla 1. Inventario del Conocimiento a Expertos en planificación urbana

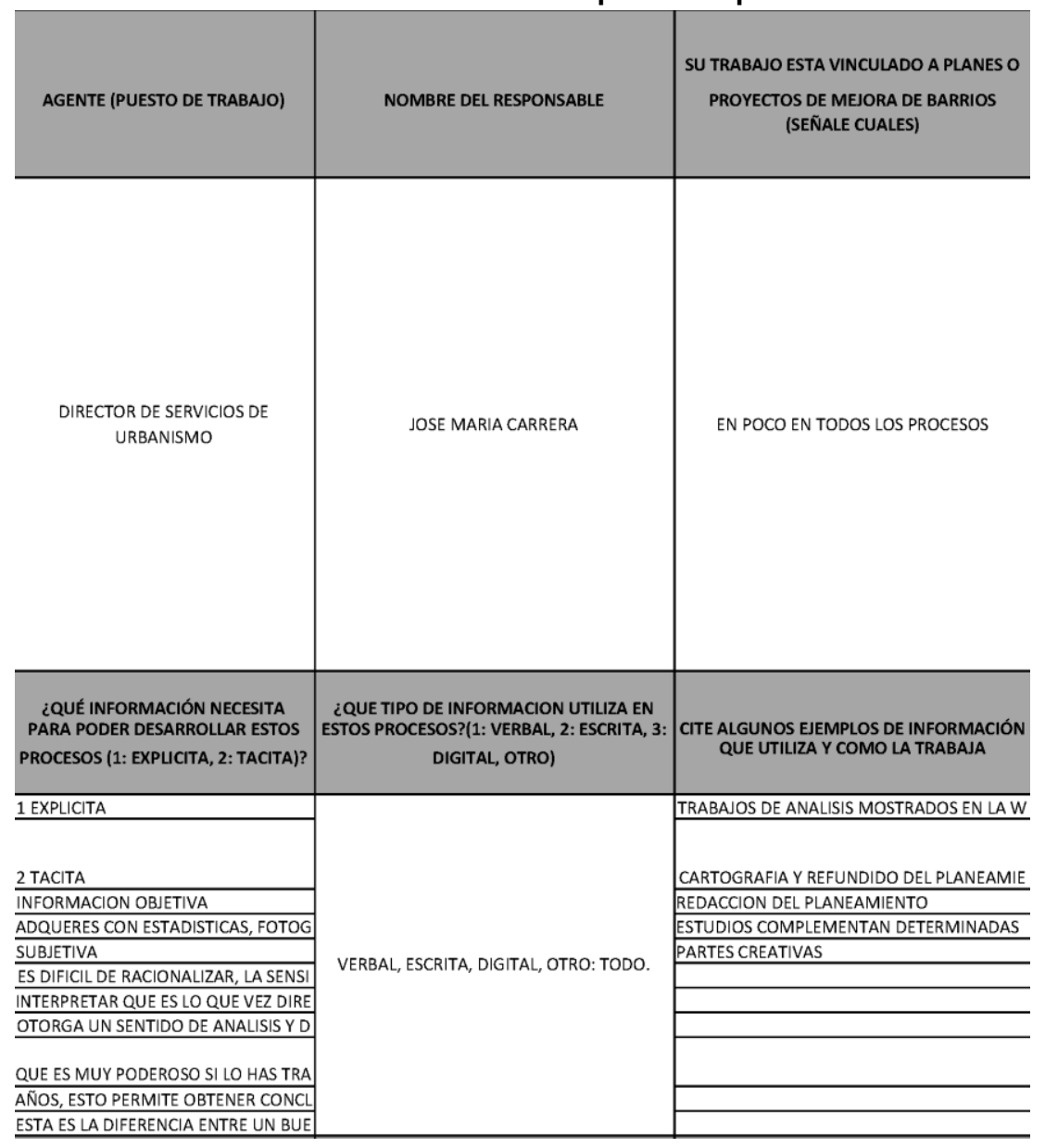

Fuente: Elaboración propia. 


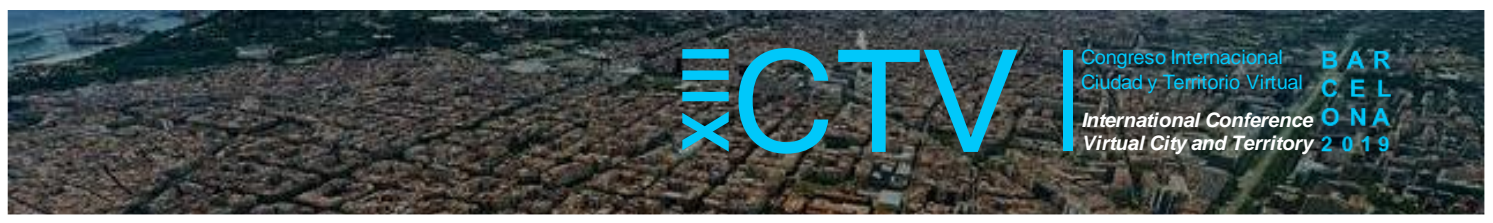

\subsubsection{Mapa del Conocimiento}

Una vez construido el inventario, la información es luego organizada en mapas de conocimiento. Un mapa del conocimiento es una representación del "modelo mental" de los conocimientos de una persona y proporciona una buena forma de conocimiento codificado, En la Figura 6 se muestra el mapa del conocimiento para el proceso de estudios previos en la elaboración del Modificación de Plan General Metropolitano de Barcelona (MPGM). En el mapa intervienen los agentes internos Dirección del Planeamiento de Urbanismo, el equipo técnico, el cual está conformado por: arquitecto de estudio de viabilidad, arquitecto de cartografía, arquitecto de validación, arquitecto de diseño de mobiliario urbano, el Departamento de Patrimonio, arquitecto de comisión de urbanismo, el arquitecto municipal, así como también, intervienen los agentes externos se encuentra Movilidad, Área Metropolitana de Barcelona (AMB), Medio Ambiente, Instituto Cartográfico de Catalunya, Consultor de Urbanismo Sostenible, Distrito de Sant Andreu, Autoritat del Transport Metropolità (ATM), Barcelona Regional, Historiadores, Asociación de Vecinos, Institutos expertas en el Barrio, Equipos de Museografía, geólogos, economistas, y biólogos, estos nodos a su vez tienen actividades bajo su responsabilidad.

El mapa tiene su punto de inicio en el nodo de estudios previos, de este nodo se desprenden una serie de conexiones a los distintos agentes internos y externos que intervienen en este proceso, de los cuales se genera conocimiento.

La mayor parte del conocimiento se genera en los agentes internos, los cuales pertenecen a la Dirección de Planeamiento del Ayuntamiento de Barcelona, pero, este proceso no podría llevarse a cabo sin el soporte de los agentes externos, los cuales tienen una labor de suministro de información para que el conocimiento pueda generarse.

En el mapa de conocimiento se puede observar que gran parte del conocimiento se genera en el nodo del Director del Proyecto, así como también, en el nodo del equipo técnico, fluyendo todo ese conocimiento a todos los nodos dependientes de este. No se observan restricciones de posible detención de flujo de conocimiento si existiera ausencia algunos de los nodos que intervienen, por lo tanto, no se encuentran huecos de conocimiento en el mapa.

Figura 6. Mapa de conocimiento del proceso de estudios previos de la MPGM

Fuente: Elaboración propia.

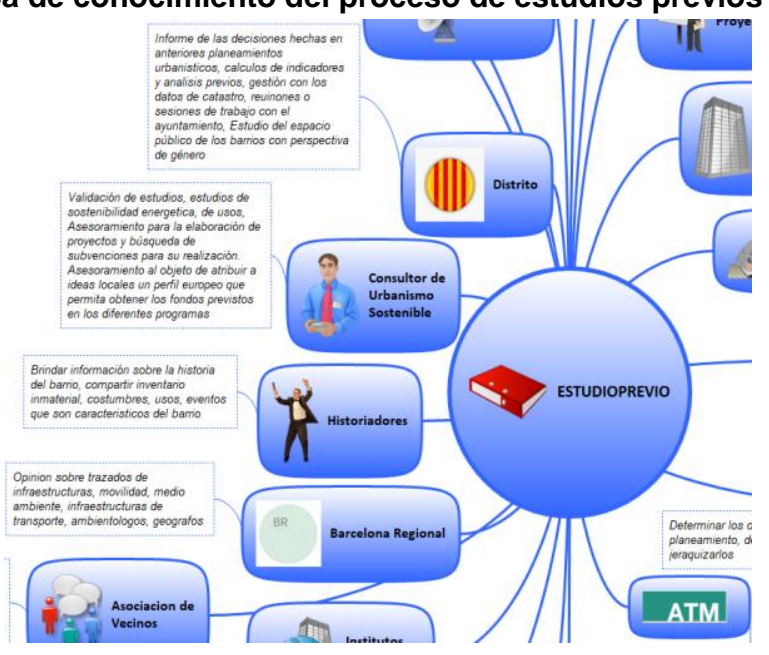




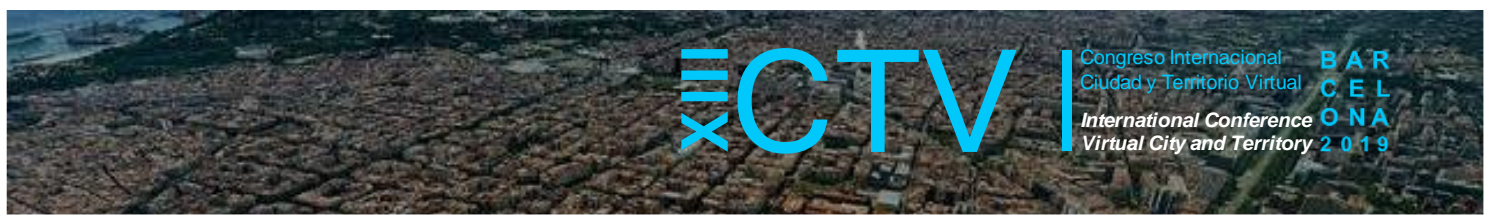

\subsubsection{Análisis de la Red Social}

Este análisis muestra los flujos de conocimiento, quién es el proveedor del conocimiento, así como, el cliente de ese conocimiento generado, sus respectivos grados de emisión, recepción, y status del conocimiento. La Figura 7 muestra el resultado obtenido para el proceso de aprobación del MPGM. Esta red social muestra de forma detallada la interacción existente en los nodos que intervienen en este proceso, se observa también, que los nodos de equipo técnico, la Dirección de Planeamiento Urbano, Distrito de Sant Andreu, prensa, subcomisión de urbanismo de Barcelona, y los letrados, son los que tienen mayor interacción, los cual significa que estos nodos son expertos en el proceso.

Los responsables del proceso es la Generalitat de Catalunya, la Subcomisión de Ecología Urbana y Movilidad y el Plenario del Consejo (grupos políticos), pero, son los nodos de la Dirección de Planeamiento Urbano, el equipo técnico, la Subcomisión de Urbanismo de Barcelona, el Distrito de Sant Andreu, y prensa los que tienen mayor grado de comunicación con los distintos agentes, los cuales tienen mayor capacidad de llegar a todos los agentes de la red.

Los nodos en color verde muestran los agentes internos o que pertenecen al departamento de urbanismo, los nodos en color amarillo los agentes externos o que no pertenecen al departamento de urbanismo y el nodo en color rojo muestra el agente responsable del proceso clave, en este caso, es el director de planeamiento de urbanismo de Barcelona.

Figura 7. Análisis de la red social para el proceso de aprobación del MPGM

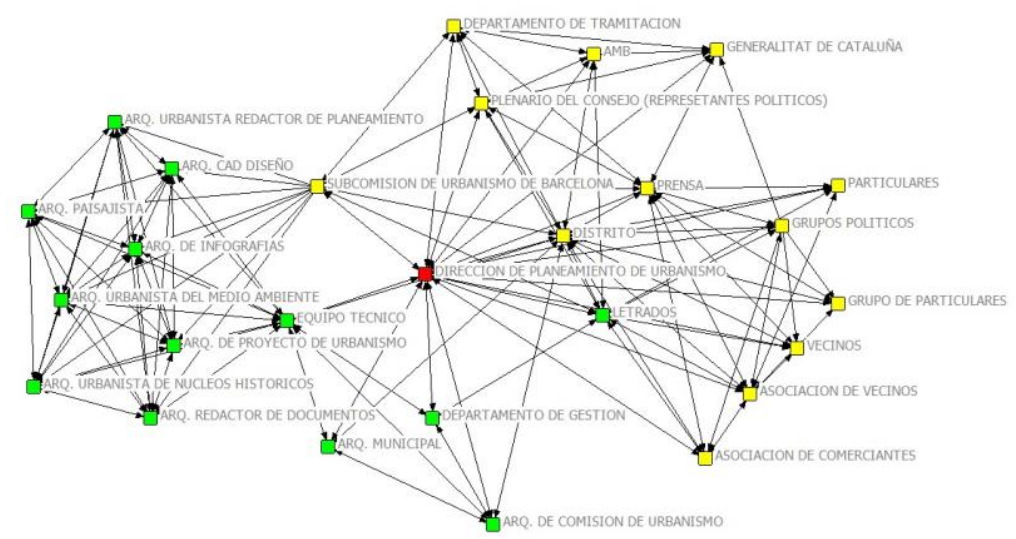

Fuente: Elaboración propia.

\subsection{Segunda Fase}

\subsubsection{Análisis FODA}

La obtención del análisis FODA se obtuvieron de los resultados obtenidos de las etapas antes mencionadas.

A continuación, en la Tabla 2 se muestra el análisis FODA para el proceso de construcción de las bases del conocimiento para la elaboración de planes y proyectos de transformación 0 mejora a escala de barrio o centros históricos. 


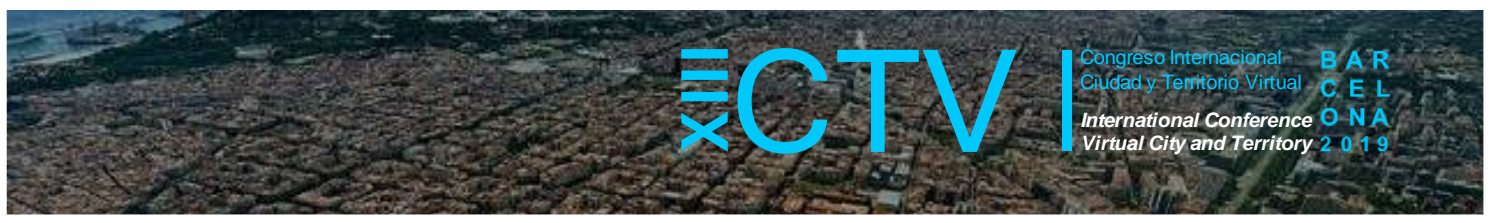

Tabla 2. Análisis FODA para el proceso de extracción del conocimiento necesario para la elaboración de los planeamientos urbanísticos a escala de barrio o mejora de centro histórico

\section{FORTALEZAS}

Se dispone de información básica en línea desde software informático Vista, Vissir, catastro, padrón, etc.

Intervención de profesionales multidisciplinares para la elaboración de planeamiento.

Accesibilidad a archivos públicos de documentación e historia del barrio.

La metodología de los procesos de elaboración de planeamiento urbanístico se encuentra estructurada.

Se concede tres meses para realización de aclaraciones, recomendaciones y reclamaciones antes de dictaminarse la aprobación final del planeamiento.

\section{OPORTUNIDADES}

Concepción del urbanismo implicando a la sociedad en un contexto sostenible.

El tema "trafico" es la parte más importante en la actualidad del planeamiento.

En ciertos proyectos la opinión que tienen los vecinos no debe tomarse en cuenta debido a las características del barrio.

Debe de dársele importancia al patrimonio inmaterial, las costumbres, eventos, actividades, que son característicos del barrio que los habitantes lo convierten en conciencia social, esta información no se encuentra digitalizada ni capturada.

Actualmente la administración se centra en las mejoras del Casco Antiguo de Sant Andreu, pero no está realizando actuaciones de rehabilitación del Casco.

\section{DEBILIDADES}

Metodología de los procesos de elaboración de planeamiento urbanístico estructurada pero no formalizada.

Las relaciones y comunicación entre la administración y las entidades vecinales es muy débil.

Mucha de las bases de datos entre el padrón y catastro no es compatible.

Difícil comunicación entre el planificador/gestor urbanístico y los habitantes del barrio a planificar debido al diferente lenguaje que utilizan.

El Ayuntamiento se planifica económicamente anualmente, y dificulta la planificación/ejecución de un proyecto que tiene una duración de 10 años, sometiéndose a cambio de Ayuntamiento, a crisis económica, etc., conformándose solo con la aprobación del proyecto y con la satisfacción de los requerimientos que tienen los vecinos.

\section{AMENAZAS}

La mala relación con un líder sindical allana el proceso de planeamiento.

Presión por parte de los vecinos, asociación de vecinos, grupos políticos, prensa, asociación de comerciantes, etc.

El factor tiempo de los agentes es impedimento para el proceso de la ejecución.

Si no publica la Comisión el resultado obtenido sobre la aprobación del planeamiento, no es vigente.

Fuente: Elaboración propia. 


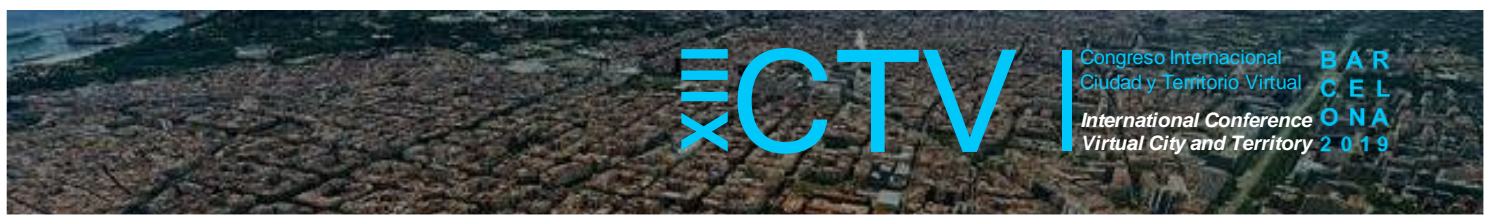

\subsection{Tercera Fase}

\subsubsection{Sistema tecnológico.}

El sistema tecnológico hace la función de ser un repositorio de la documentación y tiene la función de registrar la información obtenida durante esta estrategia, así como también, visualizar el proceso de la GC. El sistema es un software que apoya a la documentación y consulta del conocimiento que se trabaja y genera durante la elaboración de planeamiento urbanístico. El sistema permite coleccionar y publicar documentos, facilita la gestión del conocimiento generado, utilizado o recogido. Está pensado especialmente para publicar archivos o documentos en intranets corporativas o para asuntos personales.

El sistema tecnológico es una plataforma que permite la compartición de la información. Dentro de su núcleo, existe un área de trabajo, que está enfocado en realizar el deposito. Este depósito almacena la información mediante la forma de sistema de ficheros, el cual, realiza una jerarquía de archivos y directorios.

El software seleccionado para el desarrollo del sistema tecnológico para la gestión del conocimiento fue gladtolink, Gladtolink es un repositorio de documentación multiusuario o sistema basado en el conocimiento. En el sistema Gladtolink, los usuarios son capaces de capturar documentos y asignarles atributos cada vez que un documento es agregado. Otros usuarios pueden localizar los documentos usando la estructura jerarquizada de carpetas 0 utilizando el método de búsqueda integrado, La Figura 8 muestra la interface del sistema tecnológico.

Figura 8. Gestión de la documentación mediante el sistema tecnológico.

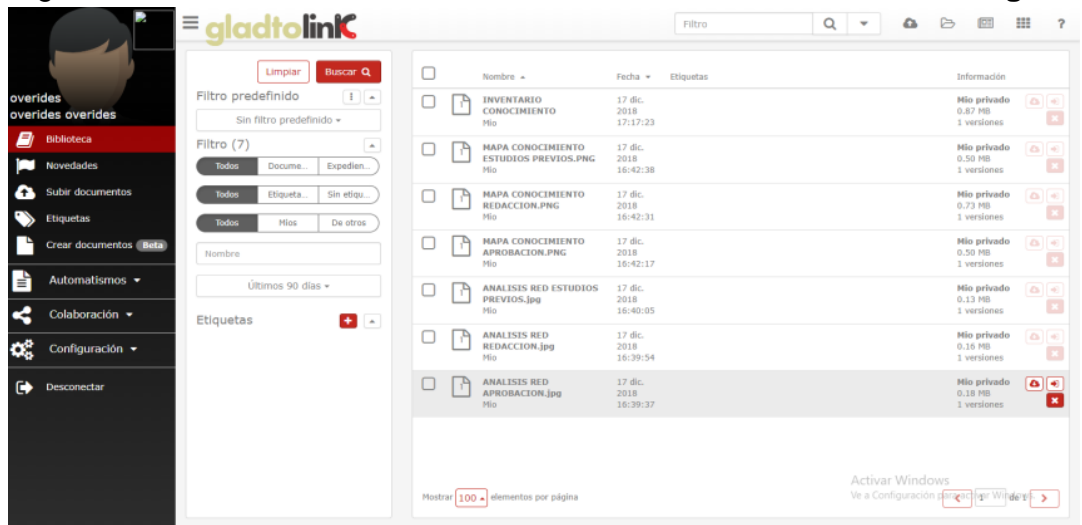

Fuente: Elaboración propia.

\section{Resultados y Discusión}

En esta etapa se concluye que los principales logros o avances en el conocimiento obtenidos en tema de sistema de GC orientando al planeamiento urbanístico, resaltan que los planificadores/gestores disponen de información básica en línea desde software informático (Vista, Vissir, catastro, padrón, etc.), pero algunos de ellos, tal como, carecen de lo social o de la percepción de los habitantes del barrio, también es posible destacar, que el factor tiempo de los agentes es impedimento para el proceso de la ejecución, algunos arquitectos, hacen 


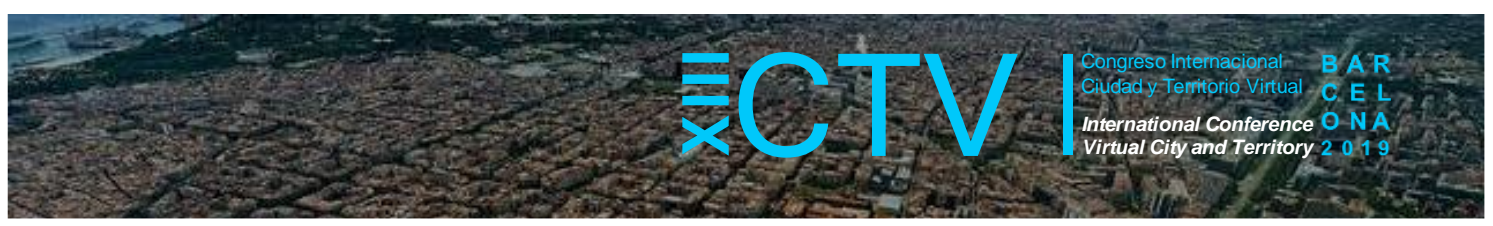

mención, que durante el proceso de elaboración de planeamiento, en algunos casos, los tiempos de ejecución del mismo, no coinciden entre los habitantes y los planificadores, también, se encontró que la mayor parte del flujo de conocimiento en los procesos de estudios previos, redacción y aprobación, fluye por los técnicos del Departamento de Urbanismo que a su vez trabajan con los agentes externos involucrados en el proceso o que no pertenecen al Departamento, actualmente, dentro del Casco Antiguo de Sant Andreu, no se realiza rehabilitación, si no, mejoras urbanas.

El análisis FODA facilitó la comprensión de las fortalezas y debilidades, ayuda a clarificar y resumir las principales cuestiones y oportunidades de la información obtenida, y esto facilita el desarrollo del pensamiento estratégico, permitió explorar eficazmente los factores positivos y neutralizar o eliminar el efecto de los factores negativos, genera ideas nuevas para ayudar a aprovechar las fortalezas. Finalmente, toda la información generada del análisis de la estrategia y del análisis FODA de la estrategia fue guardada en el sistema tecnológico para su fácil gestión y utilización de la misma.

\section{Conclusiones}

Este documento debate la relevancia de la utilización de metodologías de GC en la elaboración de planes de mejora urbana, que, aunque es un tema en auge, existe evidencia dentro del sector empresarial u organizacional que demuestra que la GC es un instrumento que aporta valor añadido en temas relacionados a este sector. Esta investigación ha mostrado el uso de la planificación estratégica colaborativa para la resolución de problemas de planificación urbana a escala de barrio o mejora de centro histórico, siendo esta de gran importancia, por lo cual, es posible avalar que las GC, debido a los resultados mostrados en este documento, que aunque su mayor aplicación en un sector con distinta problemática, pueden aportar información importante en la resolución de problemas urbanísticos, tal y como sucede con la planeación estratégica.

La GC aporta en la resolución de cuestiones urbanas mediante la participación de las organizaciones en las actividades de planificación, haciendo que el conocimiento tácito sea vinculado a los problemas de planeación con los objetivos de planificación. La identificación y elaboración de una metodología basada en GC para el análisis de parámetros físicos y urbanísticos de configuración de la ciudad, la morfología, estructura física y urbana en cuestiones relacionadas a la planificación se incorporan a un marco conceptual de GC, este marco desarrolla el análisis de variables urbanas, sociales y ambientales que influencian la percepción urbana de los habitantes del ámbito de estudio dentro de las actividades de planificación debido a que es orientado a las personas y a las iniciativas de planificación urbana, y esta, se compone por temas relacionados a la sociedad, así como por las interacciones humanas, la caracterización de que el sistema de planificación, es afectado por la idiosincrasia del ámbito a planificar, afecta a las actuaciones de GC, y que este, a su vez, se encuentra relacionado en materia de planificación urbana.

Actualmente, existe poca o casi nula bibliografía referente a la GC en la elaboración de planeamiento urbanístico, es por esto, que resulta interesante mostrar los resultados obtenidos a partir de la aplicación de la GC para el proceso de elaboración de propuestas de planificación, de transformación o de mejora de un barrio como el caso del MPGM del Casco Antiguo de Sant Andreu. 


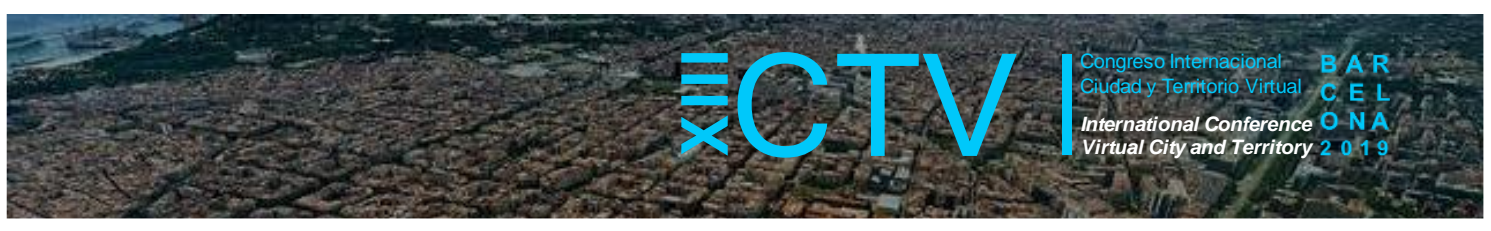

Agradecimientos: Agradecemos a los doctores Josep Roca Cladera y Pilar García Almirall por inspirarnos en esta investigación acerca planificación urbana y gestión de conocimiento.

Contribuciones de los autores: El primer autor ha desarrollado el marco de referencia, la metodología, el trabajo de campo y las conclusiones, el segundo autor ha desarrollado la metodología, el tercer autor ha desarrollado la metodología y las conclusiones, el cuarto autor ha desarrollado el trabajo de campo.

Conflicto de Intereses: No hay conflicto de intereses entre los autores.

\section{Bibliografía}

Aviv, I., Levy, M. y Hadar, I. (2009) Knowledge-Intensive business process audit: the practical aspect, Proceding of I-Know '09 and I-Semantics '09, 2(4), 397.

Baud, I. (2016). Knowledge management in urban governance; building adaptive capacity through ICT-GIS-based systems in the global South. Development, Environment and Foresight, 1(2), 7-22.

Birch, E., \& Keating, A. M. (2011). An Assessment of How Cities Create and Transfer Knowledge: A Landscape Study. Philadelphia: Penn Institute for Urban Research.

Chen, D. and Dahlman, C. (2005), The Knowledge Economy, The KAM Methodology and World Bank Operations, World Bank, Washington, DC.

Conceptdraw Mindmaps. Recuperado 9 de febrero, 2019, de https://www.conceptdraw.com/

Cooke, P. and Leydesdorff, L. (2006), "Regional development in the knowledge-based economy”, Journal of Technology Transfer, 31(1), 5-15.

Dalkir, K. (2017), "Knowledge Management in theory and practice", McGill University 1(6), 97256.

Duperet, E. (2015). Importancia de los repositorios para preservar y recuperar la información. MEDISAN, 19(10), 1-8.

Egbu C.O. and Botterill K. (2002) Information technologies for knowledge management: their usage and effectiveness, ITcon, 7, 125-136.

GladtoLink, Página de Internet, Recuperado 10 de diciembre, 2018, de http://www.gladtolink.com

Gutiérrez, H, (2005). Calidad Total y Productividad. México, DF: McGraw Hill.

Juma, C. and Yee-Cheong, L. (Eds) (2005), UN Millennium Development Library, Earthscan, London. 


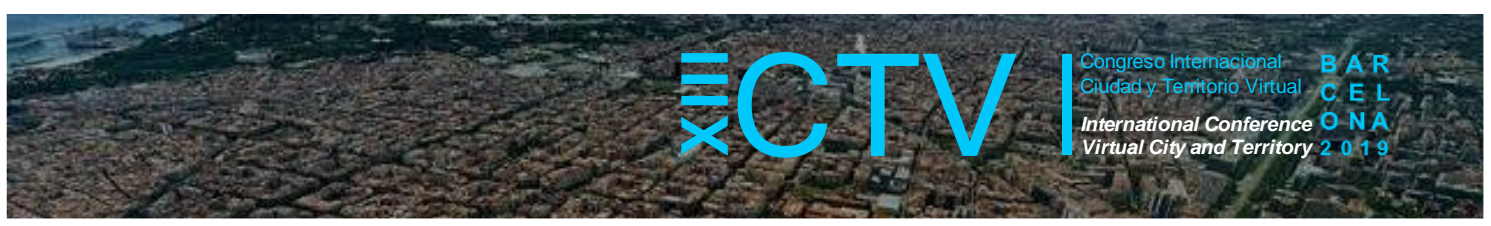

Kim S., Suh E., Hwang H. (2003). Building the knowledge map: an industrial case study, Journal of Knowledge Management, 7(2), 38-41.

Komninos, N. (2002), Intelligent Cities, Spon Press, New York, NY. Ergazakis, K., Metaxiotis, K. and Pasarras, J. (2004), "Towards knowledge cities", Journal of Knowledge Management, 8(5), 5-15.

Malizia, M. (2015). El estudio de las urbanizaciones cerradas. Una propuesta metodológica aplicada al municipio Yerba Buena (aglomerado Gran San Miguel de Tucumán, noroeste argentino). Estudios Demográficos y Urbanos, 30(1), 104-218.

Matsumoto I.T., Stapleton J., Glass J. and Thorpe T. (2005) A knowledge-capture report for multidisciplinary design environments, Journal of knowledge management, 9(3), 83-92.

Morgan, K. (2007), “The learning region”, Regional Studies, 41(1), 147-159.

Pfeffer, K., Baud, I., Denis, S., \& Sydenstricker-Neto, J. (2010). Spatial knowledge management tools in urban development. In Paper presented at 11th N-AERUS conference 2010, Bruxelles, Belgium.

Software para Gestión de Conocimiento, netdraw. Recuperado 9 de Febrero, 2019, de http://softwarekm.blogspot.com/2007/04/netdraw.html.

Strickland, A. (2001). Administración Estratégica III, 11 a Edición, México, Mc Graw Hill.

UN-HABITAT (2010), UN-HABITAT Knowledge Strategy: Building knowledge base organization, Naitobi, 7-30.

Vestal W. (2005) Knowledge mapping- the essentials for success, APQC publications Houston.

Wu Yunpeng, L., Guo Y. (2007). Knowledge Inventory Management Using Actuarial Method, Research and Practical Issues of Enterprise Information System, 2, 1487-1488. 